\title{
Randomized feasibility trial of the Scleroderma Patient-centered Intervention Network hand exercise program (SPIN-HAND): Study protocol
} (jso

\author{
Marie-Eve Carrier ${ }^{1}$, Linda Kwakkenbos ${ }^{1-3}$, Isabelle Boutron ${ }^{4,5}$, Joep Welling ${ }^{6}$, Maureen Sauve ${ }^{7,8}$, Cornelia van den Ende ${ }^{9}$, \\ Anne A. Schouffoer ${ }^{10}$, Marie Hudson ${ }^{1,11}$, Brett D. Thombs ${ }^{1,2,11-14}$, Luc Mouthon ${ }^{4,15}$, SPIN Investigators* \\ ${ }^{1}$ Lady Davis Institute of the Jewish General Hospital, Montreal, Quebec - Canada \\ ${ }^{2}$ Department of Psychiatry, McGill University, Montreal, Quebec - Canada \\ ${ }^{3}$ Behavioural Science Institute, Clinical Psychology, Radboud University, Nijmegen - The Netherlands \\ ${ }^{4}$ Université Paris Descartes, Paris - France \\ ${ }^{5}$ Assistance Publique-Hôpitaux de Paris, Paris - France \\ ${ }^{6}$ NVLE Dutch patient organization for systemic autoimmune diseases, Utrecht - The Netherlands \\ ${ }^{7}$ Scleroderma Society of Ontario, Hamilton, Ontario - Canada \\ ${ }^{8}$ Scleroderma Canada, Ottawa, Ontario - Canada \\ ${ }^{9}$ Sint Maartenskliniek, Nijmegen - The Netherlands \\ ${ }^{10}$ Leiden University Medical Center, Leiden - The Netherlands \\ ${ }^{11}$ Department of Medicine, McGill University, Montréal, Québec - Canada \\ ${ }^{12}$ Department of Epidemiology, Biostatistics, and Occupational Health, McGill University, Montréal, Québec - Canada \\ ${ }^{13}$ Department of Psychology, McGill University, Montréal, Québec - Canada \\ ${ }^{14}$ Department of Educational and Counselling Psychology, McGill University, Montréal, Québec - Canada \\ ${ }^{15}$ Service de Médecine Interne, Hôpital Cochin, Paris - France \\ * The complete list of SPIN investigators is available in the Acknowledgements section.
}

\begin{abstract}
Background: Significant functional impairment of the hands is nearly universal in systemic sclerosis (SSc, scleroderma). Hand exercises may improve hand function, but developing, testing and disseminating rehabilitation interventions in SSc is challenging. The Scleroderma Patient-centered Intervention Network (SPIN) was established to address this issue and has developed an online hand exercise program to improve hand function for SSc patients (SPIN-HAND). The aim of the proposed feasibility trial is to evaluate the feasibility of conducting a full-scale randomized controlled trial (RCT) of the SPIN-HAND intervention.

Design and methods: The SPIN-HAND feasibility trial will be conducted via the SPIN Cohort. The SPIN Cohort was developed as a framework for embedded pragmatic trials using the cohort multiple RCT design. In total, 40 English-speaking SPIN Cohort participants with at least mild hand function limitations (Cochin Hand Function Scale $\geq 3$ ) and an indicated interest in using an online hand-exercise intervention will be randomized with a 1:1 ratio to be offered to use the SPIN-HAND program or usual care for 3 months. The primary aim is to evaluate the trial implementation processes, required resources and management, scientific aspects, and participant acceptability and usage of the SPIN-HAND program.

Discussion: The SPIN-HAND exercise program is a self-help tool that may improve hand function in patients with SSc. The SPIN-HAND feasibility trial will ensure that trial methodology is robust, feasible, and consistent with trial participant expectations. The results will guide adjustments that need to be implemented before undertaking a full-scale RCT of the SPIN-HAND program.
\end{abstract}

Trial registration: ClinicalTrials.gov Identifier: NCT03092024.

Keywords: Feasibility studies, Scleroderma, Systemic, Tele-rehabilitation, Trial protocols

Accepted: September 13, 2017

Published online: October 16, 2017

Corresponding author:

Brett D. Thombs

Jewish General Hospital

4333 Côte-Sainte-Catherine Road

Montreal, Quebec

H3T 1E4, Canada

brett.thombs@mcgill.ca

\section{Introduction}

Systemic sclerosis (SSc, or scleroderma) is a rare autoimmune disease that affects the skin and internal organs including lungs, gastrointestinal tract and cardiovascular system $(1,2)$. Patient-reported problems include limitations in physical mobility and hand function, pain, fatigue, sleep disturbance, depression, sexual dysfunction, and appearance changes (3-6). Despite significant advances in symptom management, 
there is still no disease-modifying agent; thus, a primary goal of care is to reduce disability and improve health-related quality of life (HRQL) (7).

Psychosocial, educational and rehabilitation interventions can improve disease management and function, and these interventions are important components of patient-centered care in more common diseases. There are barriers to developing, testing and disseminating these interventions in rare diseases (8), and many rare disease patients, including people living with SSc, face unique challenges that are not addressed by interventions developed for more common conditions (9-12).

The Scleroderma Patient-centered Intervention Network (SPIN) was established to address this issue (8). SPIN is a collaboration of clinicians, patients and investigators from Canada, the USA, Europe and Latin America (www.spinsclero. com). Over 1900 SSc patients from over 40 centers have been enrolled in SPIN's web-based cohort. SPIN investigators are developing a series of online interventions to be tested with SPIN Cohort participants in pragmatic randomized controlled trials (RCTs).

The first SPIN intervention to be tested is an online program of hand exercises designed to improve hand function (SPIN-HAND). Contractures and deformities of the hand, consisting of decreased flexion and limited extension as well as reduced thumb abduction, are present in approximately $90 \%$ of SSc patients $(3,13)$. Impaired hand function is a major contributor to overall disability and reduced $\operatorname{HRQL}(13,14)$, and SSc patients rank impaired hand function at the top of their list of problems that impact their daily lives $(3,13)$.

Three RCTs have evaluated physical or occupational therapy interventions to improve hand function in SSc, but all were limited by small samples ( $n<20$ per arm) and significant methodological shortcomings (15). Additionally, Schouffoer et al (16) reported that a 12-week multidisciplinary day treatment program, which included hand exercises, improved grip strength and reduced disability compared to usual care 24 weeks post-randomization $(n=53)$. Recently, another RCT tested a one-month general SSc home-based exercise therapy program, which included hand exercises $(n=218)(17)$. The program improved hand function significantly at 6-months follow-up, but gains were no longer statistically significant at 12-months post-randomization. Possible reasons why the impact of the intervention on hand function was not greater and longer lasting include that it was a general exercise program that did not focus specifically on hand function and that the one-month program did not provide resources to support ongoing patient adherence. In post-trial interviews, patients encouraged the development of an internet-based tool to increase ongoing adherence and motivation (manuscript under review).

Prior to a full-scale RCT, a feasibility trial of SPIN-HAND will be conducted to ensure the feasibility of the planned trial methodology and that the online intervention is user-friendly and acceptable to trial participants (18-21). Feasibility study outcomes will inform and guide adjustments to the online intervention and the trial protocol that might need to be implemented before undertaking the full-scale SPIN-HAND RCT to assess effectiveness.

The aim of the proposed feasibility trial is to evaluate the feasibility of conducting a full-scale RCT of the SPIN-HAND intervention by obtaining data related to the study's process, required resources and management, scientific aspects, and participant acceptability. The SPIN-HAND feasibility study is not meant for hypothesis testing or effect size estimation, as the sample size is not appropriate to do so.

\section{Methods}

\section{Design and setting}

The SPIN-HAND feasibility trial will be conducted via the SPIN Cohort. The SPIN Cohort was developed as a framework for embedded pragmatic trials using the cohort multiple RCT (cmRCT) design. In the cmRCT design (22), participants enroll in an observational cohort with regular outcome measurement. Participants consent to allow their data to be used for observational studies. They consent to allow their data to be used to assess intervention trial eligibility and, if eligible, to be randomized to the intervention or control arm of the trial. They also consent that if they are eligible and randomized to the intervention arm, they will be contacted and offered access to the intervention; finally, they consent that if eligible and randomized to usual care, they will not be notified that they are involved in the trial usual care group, but their regularly collected cohort data will be used to evaluate trial outcomes. Trial eligibility will be assessed during regular SPIN Cohort assessments, which occur every 3 months, and trial outcomes will be obtained at the subsequent SPIN Cohort assessment 3 months later.

\section{Participants}

To be eligible for the SPIN Cohort, patients must be classified as having SSc based on 2013 (23) ACR/EULAR criteria confirmed by a SPIN physician, be aged $\geq 18$ years, be able to give informed consent, be fluent in English, French or Spanish, and be able to respond to questionnaires via the internet. Since this is a pragmatic trial that intends to determine if providing the SPIN-HAND Program, in addition to usual care, improves hand outcomes, patients will not be excluded if they are engaged in hand exercises as part of their usual care. The SPIN Cohort is a convenience sample. Eligible SPIN Cohort patients are recruited at SPIN sites (www.spinsclero.com/en/sites) during regular medical visits, and written informed consent is obtained. A medical data form is submitted online by the site to enroll participants. Cohort participants complete outcome measures via the internet upon enrollment and subsequently every 3 months (8).

For the SPIN-HAND feasibility trial, 40 English-speaking SPIN Cohort participants will be randomized with a 1:1 ratio to be offered to use the SPIN-HAND program or usual care, defined as the standard care that they receive from their regular healthcare providers. Cohort participants will be eligible for the feasibility trial if they complete their SPIN Cohort measures in English, have at least mild hand function limitations (Cochin Hand Function Scale (24)] [CHFS] $\geq 3$ ), and have indicated high interest in using an online hand exercise intervention ( $\geq 7$ on a $0-10$ scale). Assessment of hand function limitations and interest will occur as part of participants' regular SPIN Cohort assessments. 


\section{Procedure: randomization, allocation concealment, consent and blinding}

Randomization to be offered versus not offered, the SPIN-HAND intervention will occur at the time of Cohort participants' regular SPIN Cohort assessments. Eligible Cohort participants, based on questionnaire responses, will be randomized automatically as they complete their regular SPIN Cohort assessments using a feature in the SPIN Cohort platform, which provides immediate centralized randomization and, thus, complete allocation sequence concealment. Participants randomized to be offered the intervention will receive an automated email invitation including a link to the SPINHAND program site and the SPIN-HAND feasibility study consent form. At initial login, they will be prompted to provide written consent to participate in the SPIN-HAND feasibility study by verifying agreement with consent elements and providing their email address as the signature. Participants who consent will be automatically re-directed to the introduction page of the SPIN-HAND program. Patients who log out before agreeing to the terms of the consent form will return to the consent page upon subsequent logins. SPIN personnel will also contact participants by phone, usually within 48 hours of sending the invitation email, to describe the study, review the consent form, and answer questions. Participants who accept the offer to use the SPIN-HAND intervention can use the web link to enter the secure intervention site. Email and phone technical support will be available to help participants with the consent process and to access and use the intervention site. See Figure 1 for SPIN Cohort participants flow through the SPIN-HAND feasibility trial.

In pragmatic trials, participants are typically not blinded to intervention status and possible biases are accepted as part of the response to being offered an intervention, as may occur in practice $(25,26)$. Disappointment bias, however, can occur in conventional trial designs when a participant enrolls in a trial to receive an intervention, but is allocated to usual care $(22,25)$. For this reason, in the $\mathrm{cmRCT}$ design $(22)$, participants who are not offered an intervention are not notified that they have not been offered the intervention. This replicates actual practice, where patients are not typically advised about treatments that are not options, and reduces risk of disappointment bias $(22,26,27)$. All participants in the SPIN Cohort are aware that SPIN will conduct intervention trials and are routinely asked about potential interest in nine possible interventions, but are not informed that any particular intervention may be available unless they are offered to try the intervention. Thus, participants who are offered the intervention are not blind to their status, whereas participants assigned to usual care are blind to their participation in the trial and trial arm.

\section{Intervention}

Home-based exercise rehabilitation programs have been shown to improve hand function in SSc (17) and rheumatoid arthritis $(28,29)$. SPIN's hand exercise program is based on these programs and integrates key components of successful disease self-management programs, including goal-setting and feedback, social modeling, and mastery experiences (30-33). The SPIN-HAND exercise program was designed by

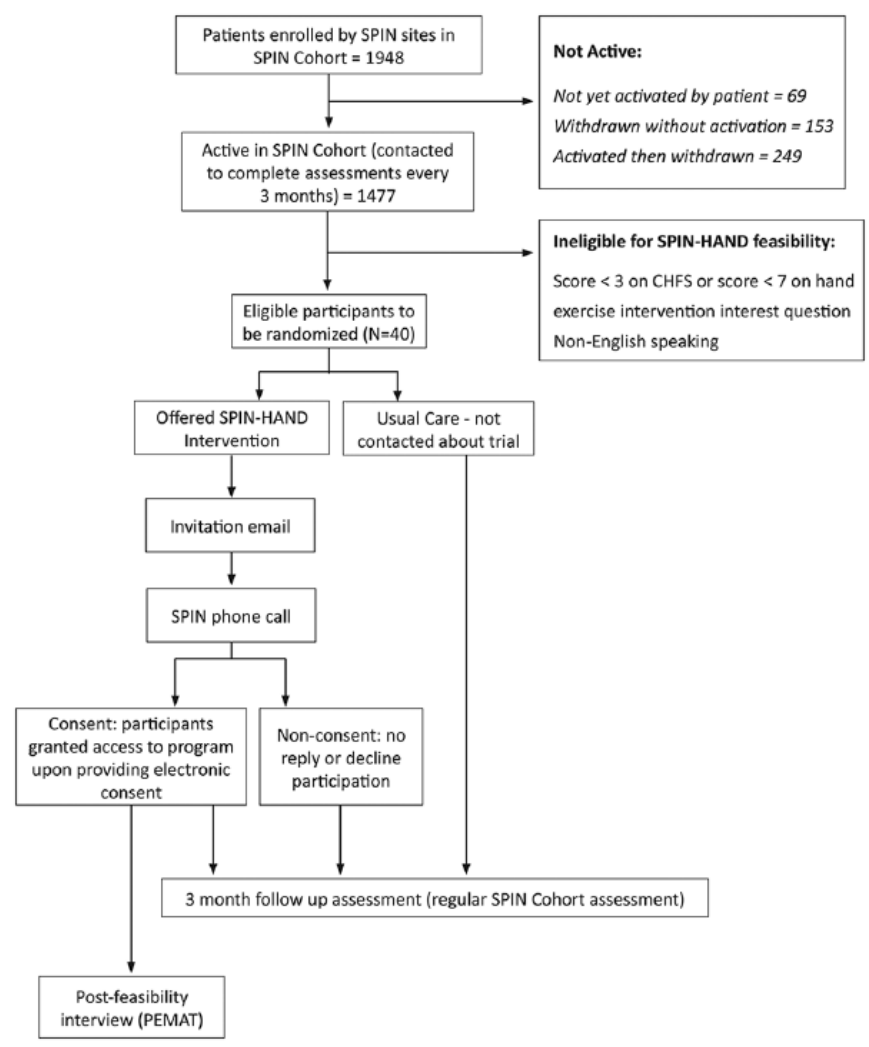

Fig. 1 - Scleroderma Patient-centered Intervention Network (SPIN) Cohort and Feasibility Trial Flow (numbers of SPIN Cohort patients as of July 24,2017$)$.

SPIN experts in physical medicine, rehabilitation, physical and occupational therapy, and behavioral therapies, together with patient representatives. The core of the program consists of four modules that address specific aspects of hand function, including (1) Thumb Flexibility and Strength (3 exercises); (2) Finger Bending (3 exercises); (3) Finger Extension (3 exercises); and (4) Wrist Flexibility and Strength (2 exercises). Participants can select the modules in the order that they prefer, based on a description of the type of function that the module is targeting to maintain or improve. The program includes sections on developing a personalized program, goal-setting strategies and examples, progress tracking, sharing goals and progress with friends and family, and patient stories of experiences with hand disability and hand exercises. The program utilizes an engaging and easy-to-navigate web interface. Instructional videos with SSc patients demonstrate and explain how to perform each exercise properly, and additional pictures illustrate common mistakes. Separate versions of each exercise are available for participants with mild-to-moderate and more severe hand involvement. In the SPIN-HAND program, participants can identify their level by reviewing photos of hands of patients with mild-to-moderate versus more severe involvement. Some exercise videos include pictures to illustrate alternate versions on how to perform the exercise when there is very severe hand involvement. Participants are provided guidance on selecting intervention intensity levels. For the first 4 weeks of the program, it is suggested that they focus on exercises in one module per week and are encouraged 
to do the exercises 3-5 times per week. Time per day graduates from 3-4 minutes in week 1, to 5-15 minutes in week 4 . Starting with week 5, participants are asked to select from a menu of program options to fit their needs and schedule. Options range from 5-10 minutes per day to 30-35 minutes per day. Participants offered the program will be able to access it for the entire 3-month trial period.

\section{Outcomes and measurement}

The primary aim of the SPIN-HAND feasibility study is to collect data related to the study's process to assess the feasibility of the steps that need to take place as part of the main study; required resources and management (e.g., personnel and data management issues); scientific aspects (e.g., outcome assessments), and participant acceptability (e.g., ease of use). Data will be used to determine whether it is feasible to carry on the main study or whether changes need to be made before undertaking a full-scale RCT. The feasibility trial outcomes related to the process and resources will be assessed throughout the duration of the feasibility trial, and participant feedback will be obtained 3 months post-randomization.

\section{Outcomes and measures}

\section{Process and resources}

Information to be collected includes: (1) the proportion of SPIN Cohort participants who meet eligibility criteria; (2) proper functioning of automated eligibility and randomization procedures; (3) the proportion of eligible participants randomized to be offered the SPIN-HAND intervention who accept the offer and consent to participate; (4) completeness of online data collection for each trial arm at 3-month follow-up; (5) completeness of the intervention usage log data; (6) ability to successfully link data coming from the SPIN Cohort and SPIN-HAND platforms; (7) rate of completion of trial outcome variables; (8) personnel requirements to call enrolled participants and help them to consent and use the SPIN-HAND program; (9) other challenges for study personnel; and (10) technological performance of the online SPIN-HAND program.

\section{Participant use and acceptability of SPIN-HAND intervention, implementation of trial procedures}

Usage of the SPIN-HAND program modules among participants in the intervention arm will be examined via intervention usage data. These data will provide detailed information on number of logins, number of modules accessed, goals set, as well as time spent on each webpage. Additionally, at 3-months' post-randomization, qualitative interviews will be conducted with participants in the intervention arm to assess user acceptability and satisfaction. The semi-structured interview will be guided by items of the Patient Education Materials Assessment Tool for Audiovisual Materials (34) and will address topics related to usability, understandability, organization, and clarity. Participant feedback from these interviews will inform any changes necessary to improve the SPIN-HAND program prior to conducting a full-scale RCT (see SPIN-HAND Intervention Patient Interviews, available online as Supplementary Material at www.sclerodermajournal.com).

Trial personnel time requirements will be logged, and trial personnel will be interviewed to understand any implementation challenges.

\section{Trial measures}

The objectives of the full-scale SPIN-HAND RCT will be to evaluate the effect of being offered access to SPIN's online hand exercise program, compared to usual care alone, on hand function (primary), functional health outcomes, and HRQL. In both the present feasibility study and the full-scale $\mathrm{RCT}$, outcome measures are routinely assessed as part of the SPIN Cohort assessments every 3 months. Thus, no additional assessments will be added for the trial that are not already done as part of the SPIN Cohort.

The 18-item CHFS (24) was developed to measure hand function limitations among patients with rheumatic diseases. The CHFS assesses ability to perform hand-related activities (e.g., kitchen, dressing oneself, hygiene, writing/typing). Items are scored on a 0-5 Likert scale $(0=$ without difficulty; 5 = impossible). Higher scores indicate less functionality. The total score is obtained by adding the scores of all items (range $0-90)$. The CHFS has good convergent validity with general functional disability measures and good sensitivity to change $(14,24,35,36)$. It has been validated in SSc (36).

Patient-reported health status will be measured using the 29-item Patient Reported Outcomes Measurement Information System (PROMIS-29) profile version 2.0. The PROMIS-29 measures seven domains of health status with four items each (physical function, anxiety, depression, fatigue, sleep disturbance, social roles and activities, pain interference) plus a single item for pain intensity. Domain items are scored on a 5-point scale (range 1-5), with different response options for different domains, whereas the single pain intensity item is measured on an 11-point rating scale. Higher scores represent more of the domain being measured; that is, better physical function and ability to participate in social roles and activities, but higher levels of anxiety, depression, fatigue, sleep disturbance, pain interference, and pain intensity. Total raw scores are obtained by summing item scores for each domain, which are converted into T-scores standardized from the general US population ( mean $=50, S D=10$ ). PROMIS-29 version 2.0 has been validated in $\operatorname{SSc}(37,38)$.

HRQL will be assessed with the EQ-5D (39), a 5-item standardized questionnaire, measuring five dimensions (mobility, self-care, usual activities, pain/discomfort, and anxiety/ depression). The items are rated from 1 (no problems) to 3 (extreme problems).

\section{Sample size}

Guidance on the appropriate sample size for feasibility trials varies substantially in the published literature, with rulesof-thumb varying from $n=12$ to $n=30$ or more per trial arm $(40,41)$. To ensure that we collect sufficient quantitative and qualitative outcome data to meet our feasibility objectives and guide the next study phase, we will include a total of 40 
SPIN Cohort participants in this feasibility trial (approximately 20 per trial arm).

\section{Data collection, storage and sharing}

Outcome measures are completed through the participants' regular SPIN Cohort assessments. The SPIN Cohort uses a secure electronic data management platform designed and managed by the Information Management Services (IMS) of the Centre for Clinical Epidemiology, Jewish General Hospital, Montreal. Separate from the SPIN Cohort portal, an encrypted database has been created for the SPIN-HAND program, which includes an identification number for intervention participants to link to SPIN Cohort data and their usage log information.

\section{Data analysis}

The primary data analysis will present a description of feasibility outcomes, including participants' eligibility and recruitment and numbers and percentages of participants who respond to follow-up measures. Use of the internet intervention will be described by presenting the frequency of logins and time spent on the SPIN-HAND program. Analysis of outcome measures will include the completeness of data and presence of floor or ceiling effects. Descriptive statistics will be used to provide means and standard deviations for the measures. Qualitative information on participants' experience using the SPIN-HAND intervention will be used to interpret acceptability related to content, webpage visuals, and navigation. Information related to required resources and management of the program during feasibility will inform any necessary changes to intervention or trial procedures.

\section{Adverse events}

The risk of adverse events occurring as a consequence of the SPIN-HAND program is very low. All hand exercises recommended are explained in detail with an emphasis on choosing a level that is comfortable for the study participant. Nonetheless, adverse events will be assessed via interview and openended questions. Any events reported will be discussed with clinical members of the team and referrals to local SPIN physicians will be made as necessary. Any serious adverse events that occur will also be reported to the ethics committee.

\section{Ethics and trial registration}

Ethics approval for the SPIN-HAND feasibility trial has been obtained from the Research Ethics Committee of the Jewish General Hospital, Montreal, Canada. The SPIN-HAND feasibility study was registered prior to participant enrollment (NCT03092024) and will be reported in accordance with standards articulated in the Consolidated Standards of Reporting Trials (CONSORT) extensions for randomized pilot and feasibility trials (21).

\section{Discussion}

The SPIN-HAND exercise program may improve hand function in SSc patients. This feasibility study will ensure that trial methodology is robust, feasible, and consistent with participant expectations (18-21). Results will guide any changes that need to be implemented before conducting a full-scale RCT to test the effectiveness of the SPIN-HAND intervention. Based on current SPIN Cohort data, we anticipate that $45 \%-50 \%$ of Cohort patients will be eligible for the full-scale SPIN-HAND trial. Thus, we anticipate being able to easily meet recruitment goals for that trial, and patients who are included in the intervention arm of the feasibility trial will not be eligible for the subsequent full-scale trial. If effective, it will be made available through patient organizations around the world to support people in their efforts to cope with living with SSc.

\section{Acknowledgements}

\section{List of SPIN investigators}

Murray Baron, McGill University, Montreal, Quebec, Canada; Susan J. Bartlett, Dan Furst, University of California, Los Angeles, California, USA; Karen Gottesman, Scleroderma Foundation, Los Angeles, California, USA; McGill University, Montreal, Quebec, Canada; Frank van den Hoogen, Radboud University Medical Center and Sint Maartenskliniek, Nijmegen, The Netherlands; Vanessa Malcarne, San Diego State University, San Diego, California, USA; Maureen D. Mayes, University of Texas McGovern School of Medicine, Houston, Texas, USA; Warren R. Nielson, St. Joseph's Health Care, London, Ontario, Canada; Robert Riggs, Scleroderma Foundation, Danvers, Massachusetts, USA; Fredrick Wigley, Johns Hopkins University School of Medicine, Baltimore, Maryland, USA; Shervin Assassi, University of Texas McGovern School of Medicine, Houston, Texas, USA; Angela Costa Maia, University of Minho, Braga, Portugal; Ghassan El-Baalbaki, Université du Québec à Montréal, Montreal, Quebec, Canada; Carolyn Ells, McGill University, Montreal, Quebec, Canada; Kim Fligelstone, Scleroderma Society, London, UK; Catherine Fortune, Scleroderma Society of Ontario, Hamilton, Ontario, Canada; Tracy Frech, University of Utah, Salt Lake City, Utah, USA; Dominique Godard, Association des Sclérodermiques de France, Sorel-Moussel, France; Daphna Harel, New York University, New York, New York, USA; Ann Impens, Midwestern University, Downers Grove, Illinois, USA; Yeona Jang, McGill University, Montreal, Quebec, Canada; Sindhu R. Johnson, Toronto Scleroderma Program, Mount Sinai Hospital, Toronto Western Hospital, and University of Toronto, Toronto, Ontario, Canada; Ann Tyrell Kennedy, Federation of European Scleroderma Associations, Dublin, Ireland; Annett Körner, McGill University, Montreal, Quebec, Canada; Maggie Larche, McMaster University, Hamilton, Ontario, Canada; Catarina Leite, University of Minho, Braga, Portugal; Carlo Marra, Memorial University, St. John's, Newfoundland, Canada; Karen Nielsen, Scleroderma Society of Ontario, Hamilton, Ontario, Canada; Janet Pope, University of Western Ontario, London, Ontario, Canada; Alexandra Portales, Asociación Española de Esclerodermia, Madrid, Spain; Tatiana Sofia Rodriguez Reyna, Instituto Nacional de Ciencias Médicas y Nutrición Salvador Zubirán, Mexico City, Mexico; Russell J. Steele, Jewish General Hospital and McGill University, Montreal, Quebec, Canada; Maria E. Suarez-Almazor, University of Texas MD Anderson Cancer Center, Houston, Texas, USA; Durhane Wong-Rieger, Canadian Organization for Rare Disorders, Toronto, Ontario, Canada; Christian Agard, Centre Hospitalier Universitaire - Hôtel-Dieu de Nantes, Nantes, France; Alexandra Albert, Université Laval, Quebec, Quebec, Canada; Marc André, Centre Hospitalier Universitaire Gabriel-Montpied, Clermont-Ferrand, France; Guylaine Arsenault, Université de Sherbrooke, 
Sherbrooke, Quebec, Canada; Nouria Benmostefa, Assistance Publique Hôpitaux de Paris - Hôpital Cochin, Paris, France; Ilham Benzida, Assistance Publique Hôpitaux de Paris - Hôpital St-Louis, Paris, France; Sabine Berthier, Centre Hospitalier Universitaire Dijon Bourgogne, Dijon, France; Lyne Bissonnette, Université de Sherbrooke, Sherbrooke, Quebec, Canada; Gilles Boire, Université de Sherbrooke, Sherbrooke, Quebec, Canada; Alessandra Bruns, Université de Sherbrooke, Sherbrooke, Quebec, Canada; Patricia Carreira, Servicio de Reumatologia del Hospital 12 de Octubre, Madrid, Spain; Marion Casadevall, Assistance Publique Hôpitaux de Paris - Hôpital Cochin, Paris, France; Benjamin Chaigne, Assistance Publique Hôpitaux de Paris - Hôpital Cochin, Paris, France; Lorinda Chung, Stanford University, Stanford, California, USA; Pascal Cohen, Assistance Publique Hôpitaux de Paris - Hôpital Cochin, Paris, France; Pierre Dagenais, Université de Sherbrooke, Sherbrooke, Quebec, Canada; Christopher Denton, Royal Free London Hospital, London, UK; Robyn Domsic, University of Pittsburgh, Pittsburgh, Pennsylvania, USA; James V. Dunne, St. Paul's Hospital and University of British Columbia, Vancouver, British Columbia, Canada; Regina Fare, Servicio de Reumatologia del Hospital 12 de Octubre, Madrid, Spain; Dominique Farge-Bancel, Assistance Publique Hôpitaux de Paris - Hôpital St-Louis, Paris, France; Paul R. Fortin, CHU de Québec - Université Laval, Quebec, Quebec, Canada; Anna Gill, Royal Free London Hospital, London, UK; Jessica Gordon, Hospital for Special Surgery, New York City, New York, USA; Brigitte Granel-Rey, Aix Marseille Université, and Assistance Publique Hôpitaux de Marseille - Hôpital Nord, Marseille, France; Claire Grange, Centre Hospitalier Lyon Sud, Lyon, France; Genevieve Gyger, Jewish General Hospital and McGill University, Montreal, Quebec, Canada; Eric Hachulla, Centre Hospitalier Régional Universitaire de Lille -Hôpital Claude Huriez, Lille, France; Pierre-Yves Hatron, Centre Hospitalier Régional Universitaire de Lille - Hôpital Claude Huriez, Lille, France; Ariane L Herrick, University of Manchester, Salford Royal NHS Foundation Trust, Manchester, UK; Adrian Hij, Assistance Publique Hôpitaux de Paris - Hôpital St-Louis, Paris, France; Monique Hinchcliff, Northwestern University, Chicago, Illinois, USA; Alena Ikic, Université Laval, Quebec, Quebec, Canada; Niall Jones, University of Alberta, Edmonton, Alberta, Canada; Artur Jose de B. Fernandes, Université de Sherbrooke, Sherbrooke, Quebec, Canada; Suzanne Kafaja, University of California, Los Angeles, California, USA; Nader Khalidi, McMaster University, Hamilton, Ontario, Canada; Benjamin Korman, Northwestern University, Chicago, Illinois, Marc Lambert, Centre Hospitalier Régional Universitaire de Lille - Hôpital Claude Huriez, Lille, France; David Launay, Centre Hospitalier Régional Universitaire de Lille - Hôpital Claude Huriez, Lille, France; USA; Patrick Liang, Université de Sherbrooke, Sherbrooke, Quebec, Canada; Jonathan London, Assistance Publique Hôpitaux de Paris - Hôpital Cochin, Paris, France; David Luna, Instituto Nacional de Ciencias Médicas y Nutrición Salvador Zubirán, Mexico City, Mexico; Joanne Manning, Salford Royal NHS Foundation Trust, Salford, UK; Maria Martin, Servicio de Reumatologia del Hospital 12 de Octubre, Madrid, Spain; Thierry Martin, Les Hôpitaux Universitaires de Strasbourg - Nouvel Hôpital Civil, Strasbourg, France; Ariel Masetto, Université de Sherbrooke, Sherbrooke, Quebec, Canada; François Maurier, Hôpitaux Privés de Metz - Hôpital Belle-Isle, Metz, France; Arsene Mekinian, Assistance Publique Hôpitaux de Paris - Hôpital St-Antoine, Paris, France; Sheila Melchor, Servicio de Reumatologia del Hospital 12 de Octubre, Madrid, Spain; Romain Paule, Assistance Publique Hôpitaux de Paris - Hôpital Cochin, Paris, France; Alexis Régent, Assistance Publique Hôpitaux de Paris - Hôpital Cochin, Paris, France; Sébastien Rivière, Assistance Publique Hôpitaux de Paris - Hôpital St-Antoine, Paris, France; David Robinson, University of Manitoba, Winnipeg, Manitoba, Canada; Esther Rodriguez, Servicio de Reumatologia del Hospital 12 de Octubre, Madrid, Spain; Sophie Roux, Université de Sherbrooke, Sherbrooke, Quebec, Canada; Perrine Smets, Centre Hospitalier Universitaire Gabriel-Montpied, $\mathrm{Cl}$ ermont-Ferrand, France; Doug Smith, University of Ottawa, Ottawa, Ontario, Canada; Vincent Sobanski, Centre Hospitalier Régional Universitaire de Lille - Hôpital Claude Huriez, Lille, France; Robert Spiera, Hospital for Special Surgery, New York, New York, USA; Virginia Steen, Georgetown University, Washington, DC, USA; Evelyn Sutton, Dalhousie University, Halifax, Nova Scotia, Canada; Benjamin Terrier, Assistance Publique Hôpitaux de Paris - Hôpital Cochin, Paris, France; Carter Thorne, Southlake Regional Health Centre, Newmarket, Ontario, Canada; John Varga, Northwestern University, Chicago, Illinois, USA; Pearce Wilcox, St. Paul's Hospital and University of British Columbia, Vancouver, British Columbia, Canada; Julie Cumin, Jewish General Hospital, Montreal, Quebec, Canada; Rina S. Fox, San Diego State University and University of California, San Diego, San Diego, California, USA; Shadi Gholizadeh, San Diego State University and University of California, San Diego, San Diego, California, USA; Lisa R. Jewett, Jewish General Hospital and McGill University, Montréal, Québec, Canada; Brooke Levis, Jewish General Hospital and McGill University, Montreal, Quebec, Canada; Sarah D. Mills, San Diego State University and University of California, San Diego, San Diego, California, USA; Mia R. Pepin, Jewish General Hospital, Montreal, Quebec, Canada; Kimberly Turner, Jewish General Hospital, Montreal, Quebec, Canada.

The SPIN team is dedicating the SPIN-HAND program to the memory of Dr. Serge Poiraudeau, who led the SPIN-HAND project team, along with Dr. Luc Mouthon. Dr. Poiraudeau cared deeply for the quality of life and well-being of people living with scleroderma, and without his leadership and dedication the SPIN-HAND program would not have been possible.

\section{Disclosures}

Financial support: SPIN, including the present feasibility trial, has been funded by grants from the Canadian Institutes of Health Research (TR3-119192, PJT-148504, PJT-149073) and the Arthritis Society. In addition, SPIN has received institutional contributions from the Lady Davis Institute for Medical Research of the Jewish General Hospital, Montreal, Canada and from McGill University, Montreal, Canada. SPIN has also received support from the Scleroderma Society of Ontario, Scleroderma Canada, and Sclérodermie Québec. Dr. Kwakkenbos was supported by a CIHR Banting Postdoctoral Fellowship. Dr. Thombs was supported by a Fonds de recherche du Québec - Santé (FRQS) researcher salary award.

Conflict of interest: None of the authors has financial interest related to this study to disclose.

\section{References}

1. Seibold J. Scleroderma. In: Harris ED, Budd RC, Firestein GS, et al, eds. Kelley's textbook of rheumatology. $7^{\text {th }}$ ed. Philadelphia, PA: Elsevier 2005; 1279-1308.

2. Wigley FM, Hummers LK. Clinical features of systemic sclerosis. In: Hochberg MC, Silman AJ, Smolen JS, et al., eds. Rheumatology. Philadelphia: Mosby 2003;1463-1480.

3. Bassel M, Hudson M, Taillefer SS, Schieir O, Baron M, Thombs BD. Frequency and impact of symptoms experienced by patients with systemic sclerosis: results from a Canadian National Survey. Rheumatology (Oxford). 2011;50(4):762-767.

4. Thombs BD, van Lankveld W, Bassel M, et al. Psychological health and well-being in systemic sclerosis: State of the science and consensus research agenda. Arthritis Care Res (Hoboken). 
2010;62(8):1181-1189.

5. Kwakkenbos L, Delisle VC, Fox RS, et al. Psychosocial aspects of scleroderma. Rheum Dis Clin North Am. 2015;41(3):519-528.

6. Jewett LR, Haythornthwaite JA, Thombs BD. Psychosocial issues and care for patients with systemic sclerosis. In: Varga J, Denton $\mathrm{CP}$, Wigley FM, eds. Scleroderma: from pathogenesis to comprehensive management. New York: Springer 2012;641-648.

7. Kowal-Bielecka O, Landewé R, Avouac J, et al. EUSTAR CoAuthors. EULAR recommendations for the treatment of systemic sclerosis: a report from the EULAR Scleroderma Trials and Research group (EUSTAR). Ann Rheum Dis. 2009;68(5):620-628.

8. Kwakkenbos L, Jewett LR, Baron M, et al. The Scleroderma Patient-centered Intervention Network (SPIN) Cohort: protocol for a cohort multiple randomised controlled trial (cmRCT) design to support trials of psychosocial and rehabilitation interventions in a rare disease context. BMJ Open. 2013;3(8):e003563.

9. Kole A, Faurisson F. The voice of 12,000 patients: experiences and expectations of rare disease patients on diagnosis and care in Europe. Available from: www.eurordis.org/IMG/ pdf/voice_12000_patients/EURORDISCARE_FULLBOOKr.pdf. Accessed September 19, 2017.

10. Huyard C. What, if anything, is specific about having a rare disorder? Patients judgements on being ill and being rare. Health Expect. 2009;12(4):361-370.

11. Nettleton S, Watt I, OMalley L, Duffey P. Understanding the narratives of people who live with medically unexplained illness. Patient Educ Couns. 2005;56(2):205-210.

12. Reimann A, Bend J, Dembski B. [Patient-centred care in rare diseases. A patient organisations perspective]. Bundesgesundheitsblatt Gesundheitsforschung Gesundheitsschutz. 2007; 50(12):1484-1493.

13. Kallen MA, Mayes MD, Kriseman YL, de Achaval SB, Cox VL, Suarez-Almazor ME. The symptom burden index: development and initial findings from use with patients with systemic sclerosis. J Rheumatol. 2010;37(8):1692-1698.

14. Rannou F, Poiraudeau S, Berezné A, et al. Assessing disability and quality of life in systemic sclerosis: construct validities of the Cochin Hand Function Scale, Health Assessment Questionnaire (HAQ), Systemic Sclerosis HAQ, and Medical Outcomes Study 36-Item Short Form Health Survey. Arthritis Rheum. 2007;57(1):94-102.

15. Poole JL. Musculoskeletal rehabilitation in the person with scleroderma. Curr Opin Rheumatol. 2010;22(2):205-212.

16. Schouffoer AA, Ninaber MK, Beaart-van de Voorde LJ, et al. Randomized comparison of a multidisciplinary team care program with usual care in patients with systemic sclerosis. Arthritis Care Res (Hoboken). 2011;63(6):909-917.

17. Rannou F, Boutron I, Mouthon L, et al. Personalized physical therapy versus usual care for patients with systemic sclerosis: a randomized controlled trial. Arthritis Care Res (Hoboken). 2017;69(7):1050-1059.

18. Kraemer HC, Mintz J, Noda A, Tinklenberg J, Yesavage JA. Caution regarding the use of pilot studies to guide power calculations for study proposals. Arch Gen Psychiatry. 2006; 63(5):484-489.

19. Thabane L, Ma J, Chu R, et al. A tutorial on pilot studies: the what, why and how. BMC Med Res Methodol. 2010;10(1):1.

20. Van Teijlingen ER, Rennie AM, Hundley V, Graham W. The importance of conducting and reporting pilot studies: the example of the Scottish Births Survey. J Adv Nurs. 2001;34(3):289-295.

21. Eldridge $\mathrm{SM}, \mathrm{Chan} \mathrm{CL}, \mathrm{Campbell} \mathrm{MJ}$, et al. PAFS consensus group. CONSORT 2010 statement: extension to randomised pilot and feasibility trials. BMJ. 2016;355:i5239.

22. Relton C, Torgerson D, OCathain A, Nicholl J. Rethinking pragmatic randomised controlled trials: introducing the cohort multiple randomised controlled trial design. BMJ. 2010;340(mar19 1): c1066.
23. van den Hoogen F, Khanna D, Fransen J, et al. 2013 classification criteria for systemic sclerosis: an American College of Rheumatology/European League against Rheumatism collaborative initiative. Arthritis Rheum. 2013;65(11):2737-2747.

24. Duruöz MT, Poiraudeau S, Fermanian J, et al. Development and validation of a rheumatoid hand functional disability scale that assesses functional handicap. J Rheumatol. 1996;23(7):1167-1172.

25. Roland $M$, Torgerson DJ. What are pragmatic trials? BMJ. 1998;316(7127):285.

26. Torgerson DJ, Torgerson CJ. Designing randomised trials in health, education, and the social sciences: an introduction. Palgrave Macmillan; 2008.

27. Zwarenstein M, Treweek S, Gagnier JJ, et al. CONSORT group; Pragmatic Trials in Healthcare (Practihc) group. Improving the reporting of pragmatic trials: an extension of the CONSORT statement. BMJ. 2008;337(nov11 2):a2390.

28. Lamb SE, Williamson EM, Heine PJ, et al. Strengthening and Stretching for Rheumatoid Arthritis of the Hand Trial (SARAH) Trial Team. Exercises to improve function of the rheumatoid hand (SARAH): a randomised controlled trial. Lancet. 2015;385(9966):421-429.

29. Foster G, Taylor SJ, Eldridge SE, Ramsay J, Griffiths CJ. Selfmanagement education programmes by lay leaders for people with chronic conditions. Cochrane Database Syst Rev. 2007;(4):CD005108.

30. Lorig KR, Holman H. Self-management education: history, definition, outcomes, and mechanisms. Ann Behav Med. 2003; 26(1):1-7.

31. Holman $\mathrm{H}$, Lorig K. Perceived self-efficacy in self-management of chronic disease. In: Schwarzer C, ed. Self-efficacy: thought control of action. Pennsylvania: Taylor \& Francis 1992.

32. Marks R, Allegrante JP, Lorig K. A review and synthesis of research evidence for self-efficacy-enhancing interventions for reducing chronic disability: implications for health education practice (part I). Health Promot Pract. 2005;6(1):37-43.

33. Marks R, Allegrante JP, Lorig K. A review and synthesis of research evidence for self-efficacy-enhancing interventions for reducing chronic disability: implications for health education practice (part II). Health Promot Pract. 2005;6(2):148-156.

34. Shoemaker SJ, Wolf MS, Brach C. Development of the Patient Education Materials Assessment Tool (PEMAT): a new measure of understandability and actionability for print and audiovisual patient information. Patient Educ Couns. 2014;96(3):395-403.

35. Poiraudeau S, Chevalier X, Conrozier T, et al. Reliability, validity, and sensitivity to change of the Cochin hand functional disability scale in hand osteoarthritis. Osteoarthritis Cartilage. 2001;9(6):570-577.

36. Brower LM, Poole JL. Reliability and validity of the Duruoz Hand Index in persons with systemic sclerosis (scleroderma). Arthritis Rheum. 2004;51(5):805-809.

37. Hinchcliff $M$, Beaumont JL, Thavarajah $\mathrm{K}$, et al. Validity of two new patient-reported outcome measures in systemic sclerosis: patient-reported outcomes measurement information system 29-item Health Profile and Functional Assessment of Chronic IIIness Therapy-Dyspnea short form. Arthritis Care Res (Hoboken). 2011;63(11):1620-1628.

38. Kwakkenbos L, Thombs BD, Khanna D, et al. Performance of the patient-reported outcomes measurement information system-29 in scleroderma: a Scleroderma Patient-centered Intervention Network Cohort Study. Rheumatology (Oxford). 2017; 56(8):1302-1311.

39. Rabin R, de Charro F. EQ-5D: a measure of health status from the EuroQol Group. Ann Med. 2001;33(5):337-343.

40. Julious SA. Sample size of 12 per group rule of thumb for a pilot study. Pharm Stat. 2005;4(4):287-291.

41. Sim J, Lewis M. The size of a pilot study for a clinical trial should be calculated in relation to considerations of precision and efficiency. J Clin Epidemiol. 2012;65(3):301-308. 